\title{
Topological Optimization of Continuum Structure based on ANSYS
}

\author{
Li Xue-ping ${ }^{1}$, Zhao Lian-yu ${ }^{1}$ and Liu Zheng-zhong ${ }^{1}$ \\ ${ }^{1}$ School of Mechanical Engineering, Tianjin Key Laboratory of the Design and Intelligent Control of the Advanced Mechanical System, \\ Tianjin University of Technology, Tianjin 300384, China
}

\begin{abstract}
Topology optimization is at the phase of structural concept design and the result of it is foundation for succeeding design, therefore, structural topology optimization is more important to engineering design. in this thesis, in order to seek the optimal structure shape of the winch's mounting bracket of ROV simulator, topology optimization design of it by finite element analysis software ANSYS was carried out. the results show that the topology optimization method is an effective optimization method and indicate that the method is correct and effective, it has a certain engineering application prospect.
\end{abstract}

\section{Introduction}

According to the type of design variables and degree of difficulty, the structural optimization of continuum structure can be divided into size optimization, shape optimization and topology optimization, corresponding to different stages of product design, namely the three stages of detailed design, basic design and concepts design. Compared to the first two optimizations, topology optimization can fundamentally change the structure of the object, the result is the basis of all the subsequent optimization of the design, so it has a great significance for engineering design; Though topology optimization has good prospects, it is recognized as the most difficult task in the field of structural optimization design.

In this study, we will use topology optimization module of ANSYS to optimize the design the winch' mounting bracket of ROV simulator and achieve the improvement of the virtual design of the winch' mounting bracket of ROV simulator, avoiding the design of blindness and improving the feasibility and effectiveness of the design, thus shortening the design cycle. We would verify the optimization results through the situation in real use. It provides an idea of simple principle taking advantage of topology optimization for design geometry element.

\section{Overview of topology optimization}

\subsection{Method for topology optimization}

In 1854, Maxwell made the basic topological analysis of the truss of minimum weight under stress constraints, laying the foundation for the study of topology optimization. In 1904, Michell put forward the theory of truss Michell [1], which veritably started the topological optimization. In 1964, Dorn and some others introduced the value theory to the field of topology optimization and proposed the Ground structural method [2] to establish a collection of nodes constituted by the structural node, the point of load application and point of support, connected with each other by member bars, and formed the socalled "ground structure" In the single load case, we would take the stress constraint into consideration, with the internal forces as design variables to build the liner programming for solution. In 1988 Bendsoe and some others proposed the homogenization method [3],which realizes the topology optimization of continuum structure by dividing the number of different micro-hole structural unit within the design area. Chinese scholars Sui Yunkang et al [4] raised the optimization model approach based on ICM (independent, continuous mapping variable) to study the topology optimization under global stress constraints, greatly reduced the number of constraints and avoided the local optimization problem. At the University of Sydney J.Lin et al [5]studied the topological optimization model of compliant mechani sms combining the advantages of SIM and PP.

Structural topology optimization theory has been proposed for more than 100 years, and it is in recent decades that it has been in rapid development, with new theoretical and computational methods emerging, being applied in various fields of mechanical, of aerospace, marine engineering and shipbuilding, etc. the topology optimization of structure is one of the effective method for the precise design of the large parts [6].

\subsection{Theory of topology optimization of Ansys}

Topology optimization theory means to seek the maximum or minimum objective function under certain constraints. The principle of topology optimization Ansys 
is to minimize the structural compliance meeting the constraints of structural volume reduction. Minimization of structural compliance is in fact a seeking of maximization of structural stiffness.

Ansys topology optimization module changes the topology optimization problem to a problem of shape optimization under a special form. The goal is to seek the maximum stiffness and the minimum volume of the structure under certain constraints, with the function of material distribution as optimization parameters. ANSYS topology optimization can be used to find the maximum stiffness, the minimum volume and maximum natural frequency of the structure. The objective function of the topology optimization reduces the structural strain energy on the condition of meeting the structural constraints. To reduce the structural strain energy means to increase the stiffness of the structure. This technology can be reached by giving density to each unit of finite element by means of design variables $\left(\eta_{i}\right)$.

Design variables assigned to each finite element (i). pseudo-density $\left(\eta_{i}\right)$. Pseudo-density value changes from 0 to $1, \eta_{i} \approx 0$ represents the remove of the material, $\eta_{i} \approx 1$ represents the preservation of the material. The total volume is the sum of volume of all the units. $V=\sum_{i} \eta_{i} V_{i}$ the pseudo-density affects the volume and elasticity tensor of each unit, $\left[E_{i}\right]=\left[E\left(\eta_{i}\right)\right]$. In the conventional linear elastic equation, the elastic tensor equals the stress and strain vector, in the formula $\left[\sigma_{i}\right]=\left[E_{i}\right]\left[\varepsilon_{i}\right],\left[\sigma_{i}\right]$ is the stress vector of the unit $i$, while $\left[\varepsilon_{i}\right]$ is its strain vector.

Taking the calculating the maximum static structural stiffness for example, under the conditions of the volume constraints, the maximum structural stiffness is to seek the minimum statistic strain energy given a certain load, which means to calculate the minimum compliance. In this case, the formula is:

$$
\left\{\begin{array}{l}
u_{c}=\min F\left(\eta_{i}\right) \\
\mathrm{s} . \mathrm{t} \int_{\Omega} \eta_{i} \mathrm{~d} \Omega \leq a V_{2}
\end{array}\right.
$$

where $u_{c}$ is the compliance; $\eta_{i}$ means the pseudo density of the unit number $i, 0 \leq \eta_{\mathrm{i}} \leq 1 ; a$ is the ratio of volume reduction; $V_{2}$ is the volume before the optimization of the structure.

When the structure receives different loading conditions, the maximum stiffness can be expressed as:

$$
F\left(U_{C}^{1}, U_{C}^{2}, \cdots U_{C}^{n}\right)=\sum_{i=1}^{n} W_{i} U_{C}^{i}, W_{i} \geq 0
$$

where $U_{c}^{i}$ is the compliance in the loading condition number $i, W_{i}$ is the component of loading conditions under the minimum stain energy.

\section{Optimization design of the winch's mounting bracket of ROV simulator}

\subsection{Description of the problem}

ROV (remotely operated underwater vehicle) is an important underwater tool of marine engineering, but the complex underwater environment leads to greater job difficulty. Taking as object a certain ROV of type openframe for observation, our research group guided by author's tutor developed 'simulator for dedicated channel of the underwater production system integration test' realizing the ROV underwater training functions. The pilot can simulate the underwater workflow and preevaluate the work program, thus reducing the risk of ROV in the actual job.

In the process of the design of ROV simulator, in order to achieve the control of front and rear pitching position, the author needs to design a mounting bracket of winch machine for fixing the winch. This paper solves this problem by means of Ansys topology optimization .

\subsection{Preprocessor before the Ansys topology optimization}

- Establish the basic structure of the topology optimization. The basic structure is the initial structure prior to the optimization. The structure should possess the features of the stress, strain etc to facilitate optimization calculations. After a comprehensive analysis of each case ultimately, the final optimal structure is determined as cuboid with a length of $1800 \mathrm{~mm}$, width of $700 \mathrm{~mm}$, and thickness $200 \mathrm{~mm}$. According to preliminary relative calculation, in extreme working conditions, winch's mounting bracket load bearing condition is shown in Fig.1, which is a schematic diagram of basic structure and stress for the topology optimization of winch mounting bracket, each of the four arrows along the $\mathrm{Y}$ axis represents horizontal shear stresses of the same value along the $\mathrm{Y}$ axis $\mathrm{F}_{\mathrm{N}}=15000 \mathrm{~N}$, while the two arrows indicate the equivalent clockwise torque of $\mathrm{X}$ axis, $\mathrm{T}_{\mathrm{N}}=1500 \mathrm{~N} . \mathrm{M}$.

- Determine the type of unit. The type of initial structure unit should be 10-node Solid 185.

- Define material properties. The selected material is304 stainless steel, elastic modulus of $1.9 \mathrm{e}+11 \mathrm{~Pa}$, Poisson's ratio of 0.29 .

- Meshing and selection of the unit 1, control the overall unit size to $0.003 \mathrm{~m}$, and then choose Quad / Free for the entire rectangular meshing.

- Apply a load to the basic structure of the model topology optimization. The finite element model of the topology optimization after the first treatment is shown in Fig. 2. 


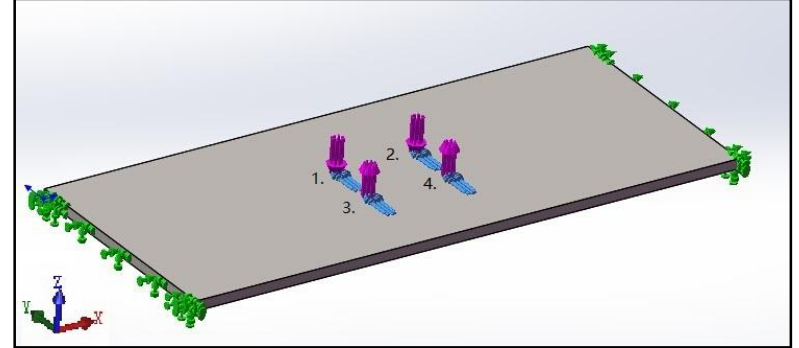

Figure 1. schematic diagram of basic structure and stress for the topology optimization

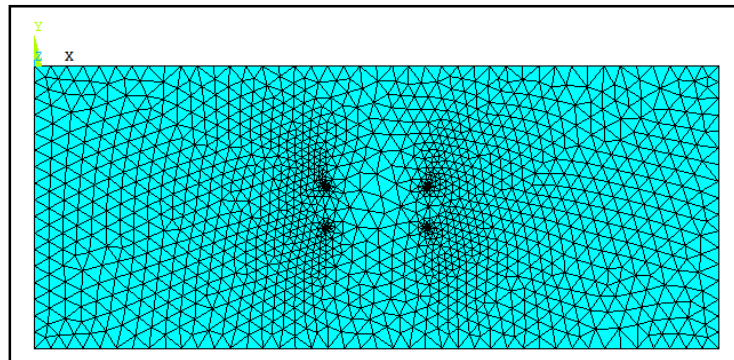

Figure 2. finite element model of the topology optimization

\subsection{Solution of topology optimization of Ansys}

- Define a single load case as the topology optimization function, the function name is Scomp.

- Define the topology optimization function Scomp as the objective function.

- Define the volume function VOLUME as the constraint function, set the reduction of volume to $60 \%$.

- Choose the method of OC(optimality criteria)for topology optimization solution, the convergence accuracy is set to 0.0001 , while the number of iterations is set to 30 .

- Run optimization program and display results

\subsection{Topology optimization results and analysis}

ANSYS topology optimization result is output as density cloud map, in fig. 3, the red section(where labeled 1) represents reserved material area, blue section (where labeled 2) represents the area of removing material.

In Fig.3, below the density of the cloud is given the contrast scale of the topology optimization design variable density value, the position of density value of 1 means the red area(where labeled 1) indicates that the structure should be arranged in structural design; while the density value of 0.001 corresponding to the blue area(where labeled 2) of density cloud map, showing that this area does not need arrangement in structural design. These are the two limiting cases, there are some color regions(where labeled 3) between the two values that need to be necessarily treated in the design process, the basic structure of the volume was significantly reduced weight of the structure is also reduced.

The objective function curves plotted course. Course objective function curve shown in Fig.4, as shown in Fig4.From the calculation process, it can be seen that structural compliance decreases with the increase of iteration, and iterative results are in stable condition after the 16th step and calculation results converge. it reflects the iterative process of structural changes in the stain energy, in an iterative process, the stain can be decreased, and gradually increases structural rigidity.

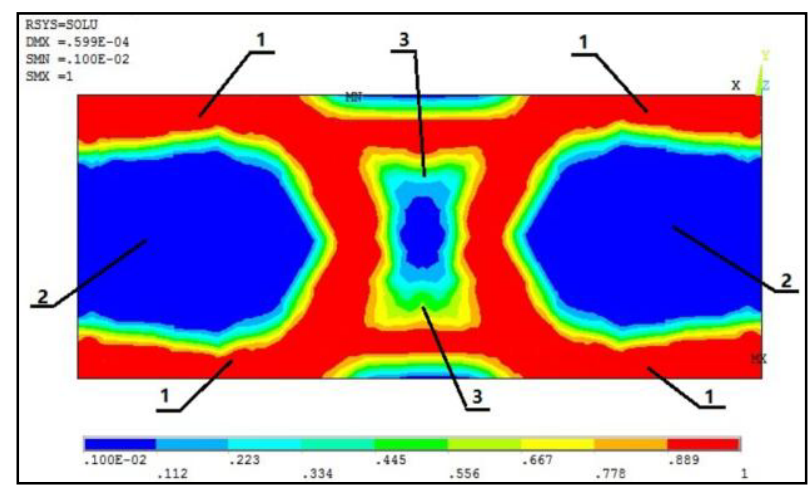

Figure 3. Pseudo-density nephogram.

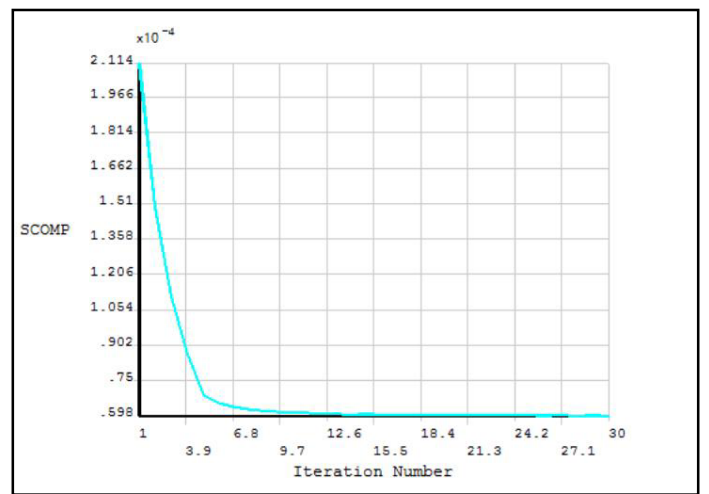

Figure 4.curves of objective function

\subsection{Design scheme of the winch's mounting bracket of ROV simulator \& Finite element Analysis}

Corresponding to pseudo density structure shown in Fig.3, core structure of the mounting bracket of winch, central area is shown in Fig.3(where labeled 1,3). This part of structure, which has greatest impact on mounting bracket's stiffness, however the optimized result in conceptual phase of design. upon most occasions it could guide the shape design of the structure, but there is still a great difference between with realistic engineering design problem the Result cannot be applied to engineering practice directly. Combine the results with the author's experience in engineering design this paper puts forward the design scheme of the winch's mounting bracket of ROV simulator as shown in Fig.5.

The core structure of the design is composed of hollow square steel tube whose cross section is $80 \mathrm{~mm} \times 80 \mathrm{~mm}$, thickness is $4 \mathrm{~mm}$ and material is 304 stainless steel,stiffened-plates are set in specific area of the structure to improve structure's stress performance. the thickness of stiffened-plate is $6 \mathrm{~mm}$ and material is 304 stainless steel.

Build the finite element model of the mounting bracket of winch with ANSYS; add material properties; apply load and boundary conditions on model; run 
static analysis program; display results as shown in fig.6, fig.7.

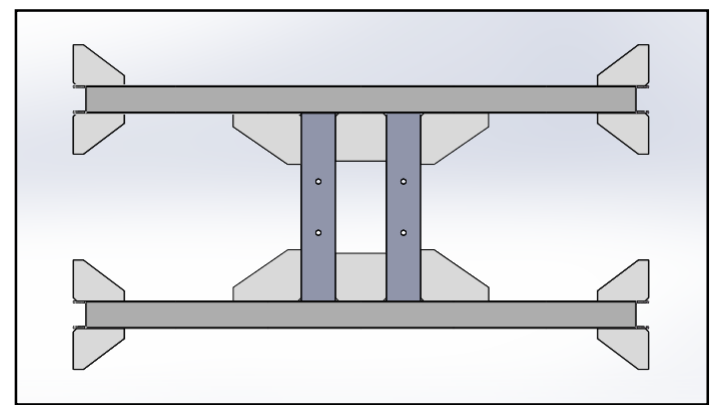

Figure 5. design scheme of the winch's mounting bracket

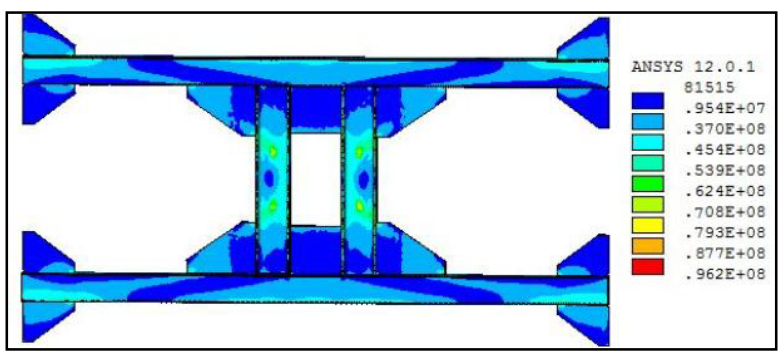

Figure 6. the stress nephogram of the winch's mounting bracket under operating limits

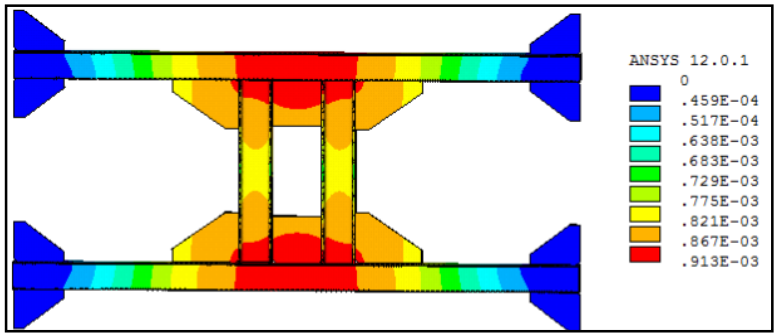

Figure 7. the displacement nephogram of the winch's mounting bracket under operating limits.

Fig. 6 shows that the maximum stress of the structure is $92.6 \mathrm{MPa}$, while the yield strength of the 304 stainless steel is $207 \mathrm{MPa}$, set the safety parameter as1.5, the permissible stress of the selected material is: $138 \mathrm{MPa}$. From the comparison between the maximum stress of the structure and the permissible stress of the selected material, it is concluded that the maximal stress of this design is under the material's allowable stress.

As shown in fig. 7 the maximum displacement of the structure is $0.913 \mathrm{~mm}$, which meet the demand of industrial design. the winch's mounting bracket of ROV simulator is produced based on topology optimization as shown in fig.8 it's function is reliable and perfect through practical testing.

It is concluded that the design of the winch's mounting bracket of ROV simulator is a great success and proved reasonable and safe.

\section{Conclusions}

- This paper expounds the mathematical processing of topological optimization of continuum structure in theory,then using the ANSYS software platform for optimization calculation. in order to realize the conceptual design.it helps to achieve stiffness and strength condition, as well as to reduce the weight of the structure reasonably and to satisfy requirement of reliability of the winch's mounting bracket of ROV simulator .

- From The results of finite element analysis and the test of winch's mounting bracket in practice, it is shown that the topology optimization model is correct and the load, the boundary constraint and the objective function is reasonable.

- Compared with traditional structural design, the structural topology optimization design method not only guarantees the reliability of the design, but also obviously shortens the design cycle, and avoid blindness and enhance the effectiveness; In addition, cost is reduced, and the topology design result has been tested in engineering application as shown in fig. 8 It is of great significance to apply the method in practice.

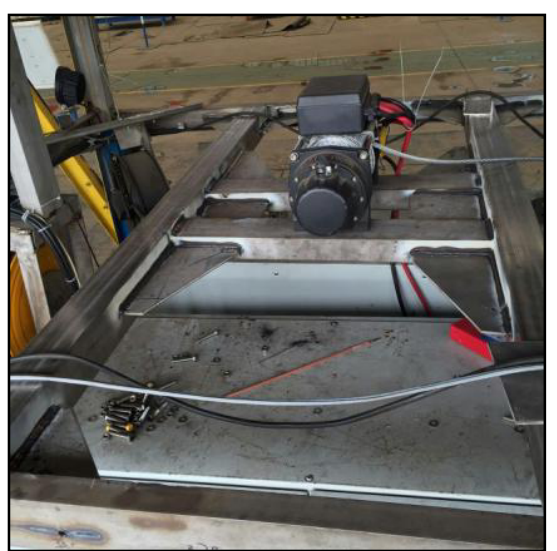

Figure 8. The winch's mounting bracket of ROV simulator produced based on topology optimization

\section{References}

1. Michell A G. The limits of economy of materials in frame structures [J]. Philosophical Magazine, 8(47),589-597(1904)

2. Dorn W S, Gomory R E, Greenberg H J. Automatic design of optimal structures[J]. J de Mechnique, 3(1),25- 52(1964)

3. Bendsoe M P,Kikuchi N. Generating optimal typologies in structural design using a homogenization method[J].Comp. Applied Mech. Engrg,71,197-224(1988)

4. SUI YK,YEHL,PENG XR. Topological optimization of continuum structure with global stress constraints based on ICM method[J].Computational Methods, PTS1-2,1003-1014(2006)

5. Lin Jiang-zi,Luo Zhen,Tong Li-yong. A new muftiobjective programming scheme for topology optimization of compliant mechanisms[J].Structural and Mufti-disciplinary Optimization,40(1-6),24125(2010)

6. Zhu Deng-lin, Chen Jun-wei, Yu Jie etc. Developments of Structural Topology Design and its Application in Structural Design [J]. Machine Building and Automation,34(6),7-11(2005) 